\title{
Article \\ CFD-DEM Simulation of Backflow Blockage of Deep-Sea Multistage Pump
}

\author{
Qiong Hu ${ }^{1,2}$, Jun Chen ${ }^{1,2}$, Liwen Deng ${ }^{1,2, *}$, Yajuan Kang ${ }^{3}$ and Shaojun Liu ${ }^{1,2,4}$ \\ 1 School of Mechanical and Electrical Engineering, Central South University, Changsha 410083, China; \\ huqiong@csu.edu.cn (Q.H.); 193712164@csu.edu.cn (J.C.); liushaojun@csu.edu.cn (S.L.) \\ 2 State Key Laboratory of Deep Sea Mineral Resources Development and Utilization Technology, \\ Changsha 410012, China \\ 3 School of Mechatronics Engineering and Automation, Foshan University, Foshan 528000, China; \\ 163701035@csu.edu.cn \\ 4 Shenzhen Research Institute, Central South University, Shenzhen 518057, China \\ * Correspondence: livedeng@csu.edu.cn; Tel.: +86-138-802-80823
}

Citation: $\mathrm{Hu}, \mathrm{Q} . ;$ Chen, J.; Deng, L.; Kang, Y.; Liu, S. CFD-DEM

Simulation of Backflow Blockage of Deep-Sea Multistage Pump. J. Mar.

Sci. Eng. 2021, 9, 987. https:// doi.org/10.3390/jmse9090987

Academic Editors: Kamal Djidjeli and Zhe Sun

Received: 19 August 2021

Accepted: 8 September 2021

Published: 10 September 2021

Publisher's Note: MDPI stays neutral with regard to jurisdictional claims in published maps and institutional affiliations.

Copyright: (c) 2021 by the authors. Licensee MDPI, Basel, Switzerland. This article is an open access article distributed under the terms and conditions of the Creative Commons Attribution (CC BY) license (https:// creativecommons.org/licenses/by/ $4.0 /)$.

\begin{abstract}
The multistage centrifugal pump is the critical component of mineral resources lifting in deep-sea mining. The reflux of nodules in the lifting pipe caused by the emergency pump stop can easily cause the pump to clog. In this paper, coupled Computational Fluid Dynamics and Discrete Element Method (CFD-DEM co-simulations) are used to clarify the solid-liquid two-phase flow in two-stage centrifugal pumps under different particle sizes (10-20, 20-30, 30-40, 40-50 mm) with constant particle concentration. The movement and accumulation behaviour of particles in different flow fields (pipeline to pump, the first to the second pump stage) is investigated. Meanwhile, the effect of particle size and particle reflux velocity on the blockage of the flow channel in the pump was investigated. Particle accumulation in the pump was observed to determine the key factors affecting the pump's reflux capacity. The residual mass of particles in the pump at different particle sizes was counted. Simultaneously, the percentage of residual mass of 10-20 mm particles in the pump was compared between the experiment and the simulation with an acceptable tolerance of within $10 \%$. In addition, pressure changes in the blockage-prone section were also investigated. A comparison between experiments and simulations verifies the consistency of the trend on the pump inlet pressure when clogged with $50 \mathrm{~mm}$ particles. It was found that larger particles in the range of $10-30 \mathrm{~mm}$ can better ensure the pump's reflux performance.
\end{abstract}

Keywords: deep-sea mining; multiple-stage centrifugal pump; reflux; CFD-DEM numerical simulation

\section{Introduction}

With the development of China's marine industry, the management and sharing of marine and ocean resources face profound changes. The deep sea is abundant in mineral resources that are scarce on land, such as manganese nodules, cobalt-rich crusts, and polymetallic sulphides [1]. With the consumption of mineral resources on land and the increasing demand for production, consensus is gradually being reached among countries on the exploitation of marine resources [2,3]. Against such a background, deep-sea mining is proposed accordingly. Hence, a series of technological developments have been carried out by various countries.

In the 1990s, China began to research deep-sea mining technologies. During the 11th Five-Year Plan, a two-stage deep-sea lifting pump was designed and manufactured. A series of experiments based on this pump was carried out. In terms of theoretical research, the solid-liquid two-phase flow mechanism of the pump has been investigated by Zou [4]. The theory provides a theoretical basis for designing the flow channel of the deep-sea lifting pump. A series of studies on fluid velocity, concentration and pressure 
has also been carried out to obtain the pump's characteristic curve. At the same time, a two-stage lifting pump was developed by Changsha Mining and Metallurgical Institute Co., Ltd. "Changsha Mining and Metallurgical Institute Co., Ltd." is a well known research institute in Changsha, China [5]. However, a blockage of particles occurred in the pump's internal flow channel when reflux containing a quantity of $50 \mathrm{~mm}$ particles was carried out. Therefore, relevant numerical simulation studies on such extreme situations are required before the experiments. Numerical simulations are used to clarify the safety thresholds for the particle size and concentration of the test particles and provide the relevant theoretical recommendations for the regular operation of the multistage centrifugal pump.

In Computational Fluid Dynamics (CFD) for centrifugal pumps, Plua and Hidalgo [6] investigated the suitability of the simulation for most pumps under the N-S equation by comparing experimental data with CFD simulation data. The following relevant conclusion was drawn: the Reynolds average Navier-Stokes (RANS) turbulence model can meet the needs of most numerical pump simulations. Huang [7] analysed the effect of the trailing edge of the blades on pressure fluctuations in the pump with CFD simulations. Zeng and Chen [8] used the mixture multiphase flow model. The numerical simulation of the solid-liquid two-phase flow in the lifting pump was carried out in their paper. The effect of particle size on the volumetric concentration distribution of the accumulation in the pump was compared. In addition, the hydraulic performance of lifting pump with different particle sizes was also investigated to reveal the effect of particle sizes on pump characteristics.

By studying the lifting pump's hydraulic properties problem, the governing equation of the homogenous slurries two-phase flow model was presented by Zou and Lu [4]. The two-phase flow model of homogenate was used by Dong [9] to divide the particle size. This homogeneous slurry model greatly facilitated the investigation of solid-liquid two-phase flow in deep-sea mining pumps. However, the DPM method is still used in this method. In this model, tiny particles are mixed with seawater as a homogenate slurry. The effect of particle action on the flow field is still neglected. The volume effect between particles and particle convection disturbance needs to be considered for the actual centrifugal pump of deep-sea mining. However, the accumulation, aggregation and collision of particles in the pump cannot be truly reflected by the DPM method. Cundall [10] proposed the discrete element method (DEM) based on the Lagrangian method, which is widely used for the calculation of multiphase flows of particles and fluids [11,12].

The coupling method of CFD and DEM can be applied to calculate the behaviour of particles in the flow field with excellent accuracy [13,14]. Li [15] analysed the activity of single particle and their applications under different operating conditions by using the CFD-DEM method to model the solid-liquid two-phase of the pump, which provides the grid feasibility for implementing the coupled CFD-DEM method of the pump. Deng [16] investigated the phenomenon of inhomogeneous local agglomeration of particles within a six-stage centrifugal pump by means of CFD-DEM. Li [17] investigated the problem of particle backflow blockage in single-stage centrifugal pump and elucidated the effect of particle size on blockage in the pump.

At present, all the work has provided strong support for the study of solid-liquid two-phase flow in deep-sea mining centrifugal pump, and the blockage mechanism of the slurry containing coarse particles in the pump is the focus of this study. CFD-DEM coupling simulation was used to simulate the backflow of coarse particles during pump shut down, considering the accumulation, condensation, and collision between particles. The coupled simulation demonstrates the blockage of particles in the pump with different particle sizes and different particle ratios. Meanwhile, the movement of the particles in the pump and the residual mass of the particles in the pump in the case of blockage as well as the pressure variation in the area prone to blockage are analysed. Based on the above research, some suggestions have been proposed to prevent the blockage of particle reflux that can potentially occur in multistage centrifugal pumps during emergency shutdown. 


\section{Structure and Mesh of Multistage Centrifugal Pump}

Six-stage guide vane lift pumps are used as the central core of the lift system to ensure the high head, significant flow rates and axial flow characteristics required by design. Figure 1 shows the structure diagram of the six-stage lifting electric pump and the overall diagram of the motor shell's annular flow channel. The six-stage lifting pump has a highspeed ratio and belongs to the segmented type's vertical multistage pump. The submersible motor was filled with liquid, and the motor's outer cylinder was used to ensure sufficient compressive capacity to overcome the high-pressure environment.
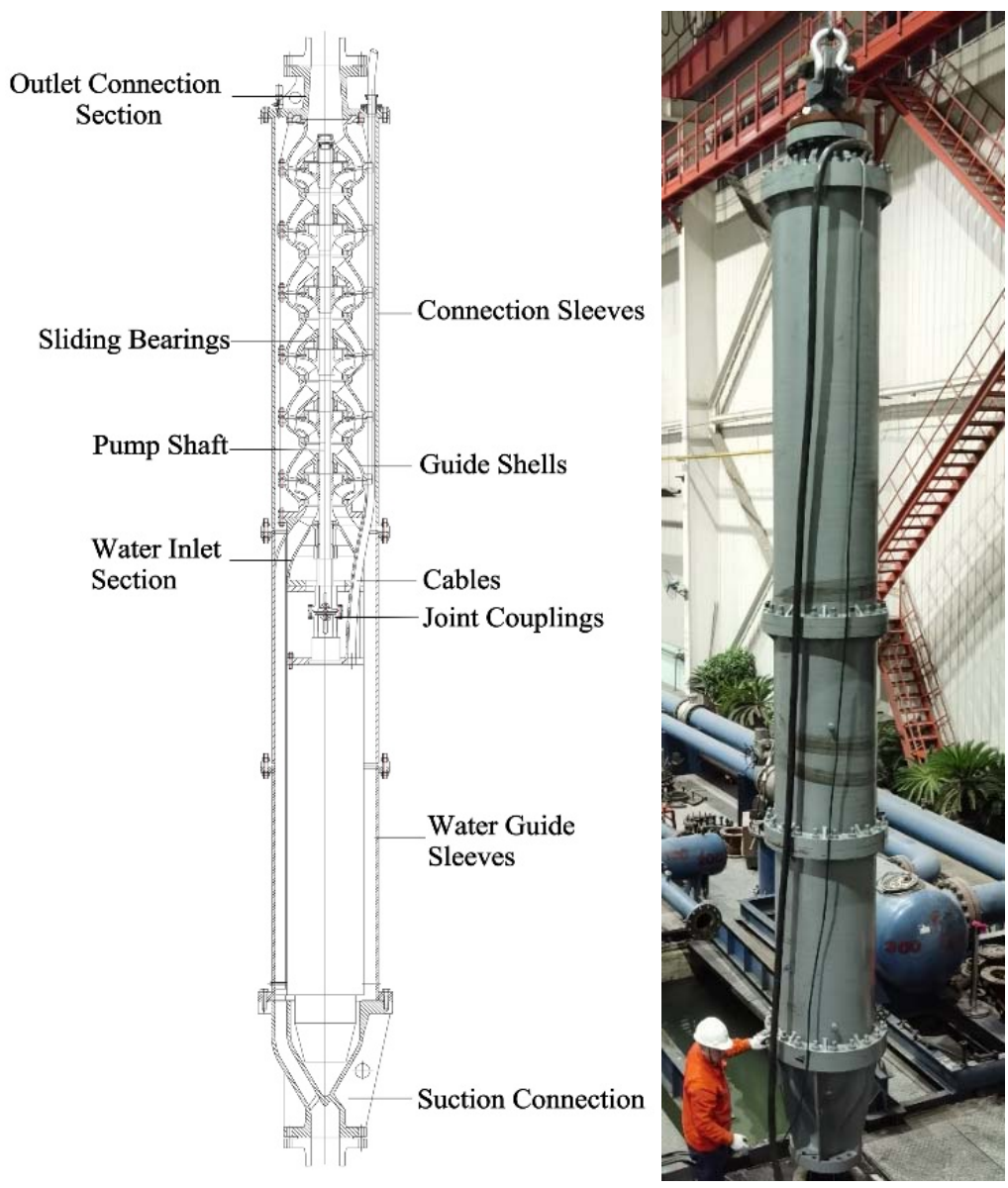

Figure 1. Overall view of the electric pump.

Given the requirement of the lifting pump for better overflow capacity, the flow path of the guide vane lifting pump was designed with the amplified flow rate method to match the channel of the coarse particle mixture slurry [18]. Figure 2 shows the section structure of the deep-sea lifting electric pump. The electric pump's standard operation mode is to suck the coarse particle mixed slurry into the lifting pump through the inlet throttling channel through the impeller's high-speed rotation and then turn the high-speed mixed slurry into the high-pressure head mixed slurry through the guide vane flow channel of the pump. Eventually, the mixed slurry is conveyed to the ore collection bin on the ship's surface through the lifting pipe. 


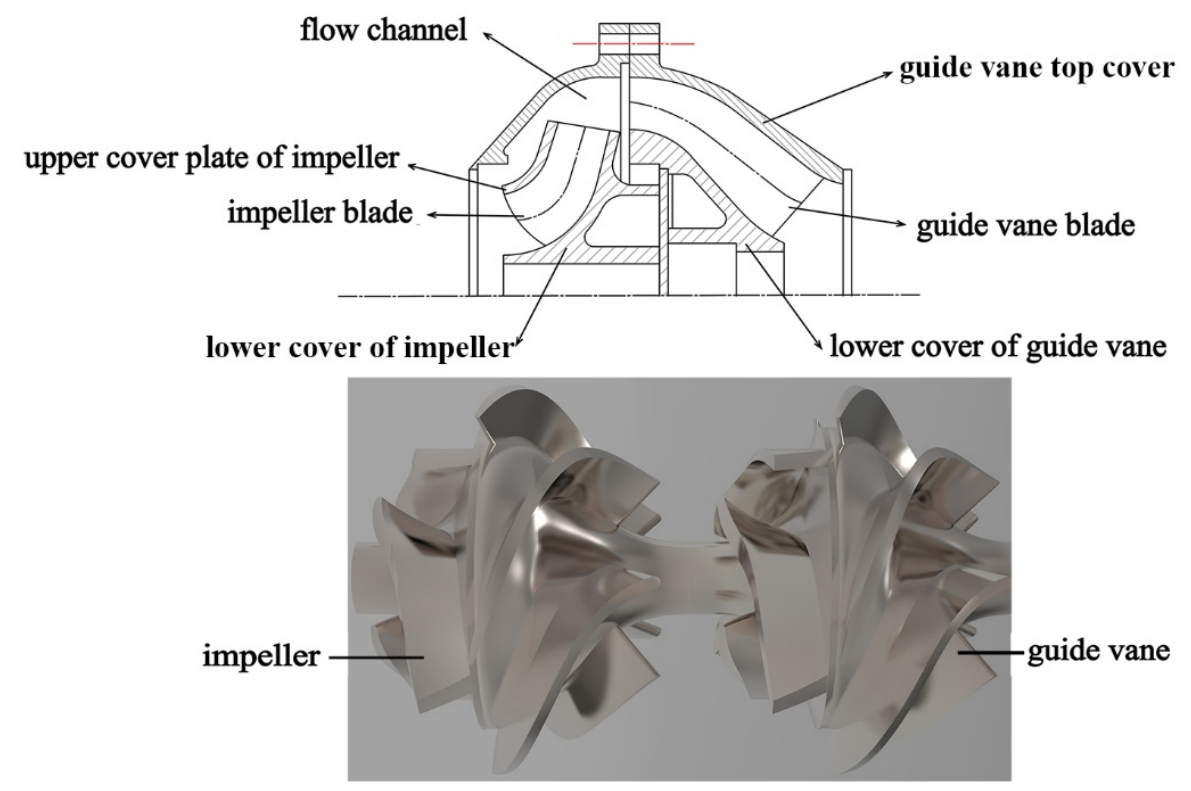

Figure 2. Deep-sea lifting electric pump structure.

The main parameters of the lifting pump system are as follows: the flow rate should reach $420 \mathrm{~m}^{3} / \mathrm{h}$, the total head of the six-stage pump should reach $270 \mathrm{~m}$, the motor speed at rated operation condition should be $1450 \mathrm{rpm}$, and the efficiency of the pump should be $52 \%$. Some key parameters of the impeller and guide vane are shown in Table 1 . Considering the safety and efficiency of sea trial systems, the particle size and concentration needed reasonable selection. The test pump's maximum particle size and pipeline transportation are determined to be $20 \mathrm{~mm}$ to reduce sea trials' technical risk. However, the study of particle reflux in the pump under the stopped status is not comprehensive enough, and further theoretical support is needed. Therefore, the authors used particle size classification to study the dynamic process of particle blockage in the flow channel and analysed the blockage causes.

Table 1. Main design parameters of the impeller.

\begin{tabular}{cccc}
\hline Structure of Impeller & Parameter & Structure of Guide Vane & Parameter \\
\hline Number of blades & 4 & Number of blades & 5 \\
Inlet diameter & $235 \mathrm{~mm}$ & Inlet diameter & $395-516 \mathrm{~mm}$ \\
Outside diameter & $395-425 \mathrm{~mm}$ & Outside diameter & $95-260$ \\
Outlet width & $60 \mathrm{~mm}$ & Outlet width & $60 \mathrm{~mm}$ \\
Inlet installing angle & $35^{\circ}$ & Inlet installing angle & $12^{\circ}$ \\
Outlet installing angle & $32.5^{\circ}$ & Outlet installing angle & $85^{\circ}$ \\
Blade angle & $110^{\circ}$ & Blade wrap angle & $95.5^{\circ}$ \\
\hline
\end{tabular}

\section{Numerical Modelling}

\subsection{Fluid Phase Control Equation}

The most suitable computational model should be selected from many computational models to describe turbulent flow correctly. Regarding the design considerations and the working conditions of large flow, the RNG $k$ - $\varepsilon$ model $[19,20]$ was selected to obtain more accurate results. The RNG $k-\varepsilon$ model is still used for the numerical simulation of the reflux field to maintain the uniformity of the computational model in the simulation. 
The constraint equation of turbulence kinetic energy $k$ and turbulence dissipation rate $\varepsilon$ is as follows [21]:

$$
\begin{gathered}
\frac{\partial}{\partial t}(\rho k)+\frac{\partial}{\partial x_{i}}\left(\rho k u_{i}\right)=\frac{\partial}{\partial x_{j}}\left(\alpha_{k} \mu_{e f f} \frac{\partial k}{\partial x_{j}}\right)+G_{k}+G_{b}-\rho \varepsilon-Y_{M}+S_{k} \\
\frac{\partial}{\partial t}(\rho \varepsilon)+\frac{\partial}{\partial x_{i}}\left(\rho \varepsilon u_{i}\right)=\frac{\partial}{\partial x_{j}}\left(\alpha_{\varepsilon} \mu_{e f f} \frac{\partial \varepsilon}{\partial x_{j}}\right)+C_{1 \varepsilon} \frac{\varepsilon}{k}\left(G_{k}+C_{3 \varepsilon} G_{b}\right)-C_{2 \varepsilon} \rho \frac{\varepsilon^{2}}{k}-R_{\varepsilon}+S_{\varepsilon}
\end{gathered}
$$

where $k$ is turbulent momentum, $\varepsilon$ turbulent dissipation rate, $\rho$ is the density of the liquid, $u_{i}$ is the direction of liquid flow, $x_{i}$ and $x_{j}$ are direction, $G_{k}$ represents the generation of turbulence kinetic energy due to the mean velocity gradients, $G_{b}$ is the generation of turbulence kinetic energy, $Y_{M}$ represents the contribution of the fluctuating dilatation incompressible turbulence to the overall dissipation rate, and $S_{k}$ and $S_{\varepsilon}$ are source terms.

According to Chen [22]:

$$
\begin{gathered}
R_{\varepsilon}=\frac{C_{\mu} \rho\left(1-\frac{\eta}{\eta_{0}}\right)}{1+\beta \eta^{3}} \frac{\varepsilon^{2}}{k}, \eta=S_{k} / \varepsilon, \beta=0.012 \\
C_{1 \varepsilon}=1.42, C_{2 \varepsilon}=1.68, C_{3 \varepsilon}=0.0845
\end{gathered}
$$

\subsection{Particle Phase Control Equations}

A more comprehensive study of multiphase flows is made, considering the interaction forces between particles and the slippage between solid and liquid phases.

Particle phase control equations for coarse particle-liquid two-phase flow [23]:

$$
\begin{gathered}
m_{i} \frac{d v_{i}}{d t}=\sum_{j=1}^{k_{i}}\left(F_{n, i j}+F_{t, i j}\right)+F_{f p, i}+G+F_{S}+F_{D}+F_{M} \\
I_{i} \frac{d w_{i}}{d t}=\sum_{j=1}^{k_{i}}\left(M_{t, i j}+M_{R, i j}\right)
\end{gathered}
$$

where $M_{i}$ and $I_{i}$ are the mass and moment of the particle $i ; v_{i}$ and $w_{i}$ are the translational and rotational velocity of the particle $i$; and $F_{n, i j}, F_{t, i j}$ are normal and tangential contact force between particles $i$ and $j$.

$F_{f p, i}$ is the interaction forces between continuous and discrete phases;

$F_{S}$ is a lift force due to fluid shear;

$F_{D}$ is the fluid drag force on the particle;

$F_{M}$ is a lift force generated by the rotation of particles in the flow field; and $M_{t, i j}, M_{r, i j}$ are tangential and rolling frictional torques acting on the particles $i$ and $j$.

The relevant formula is as follows:

$$
\begin{gathered}
F_{n, i j}=\frac{4}{3} E^{*}(R)^{\frac{1}{2}} \alpha^{\frac{3}{2}} \\
F_{t, i j}=-2 \sqrt{\frac{5}{6}} \frac{\ln e}{\sqrt{l n^{2} e+\pi^{2}}} \sqrt{G^{*} \frac{m_{p}}{2}\left(\frac{m_{p}}{2} \alpha\right)^{1 / 2} v_{t}^{r e l}} \\
G=m g \\
F_{S}=1.615 d_{p}^{2}\left(\mu_{f} \rho_{f}\right)^{\frac{1}{2}} \cdot C_{s a f f}\left|w_{c}\right|^{-\frac{1}{2}}\left(u-u_{p}\right) w_{c} \\
F_{D}=\frac{1}{8} \pi C_{D} \rho d^{2}\left|u-u_{p}\right|\left(u-u_{p}\right) \\
C_{D}=\left\{\begin{array}{c}
\frac{24}{R_{e p}}\left(1+0.15 R_{e_{p}} 0.687\right), R_{e_{p}} \leq 10^{3} \\
0.44, R_{e_{p}}>10^{3}
\end{array}\right.
\end{gathered}
$$




$$
F_{M}=\frac{1}{2} A_{p} C_{M a g n} \rho_{f} \frac{|V|}{|\Omega|}(V \times \Omega)
$$

where $E^{*}$ is the equivalent Young's modulus, $R$ is the diameter of particles, $G^{*}$ is the equivalent Shear modulus, $\alpha$ is the normal overlap distance between particles, $v_{t}^{r e l}$ is the tangential component of relative velocity between contacting particles, $C_{D}$ is the coefficient of drag force, $\rho$ is the density of the fluid, $\mu_{f}$ is the liquid's viscosity, $d$ is particle diameter, $u_{f}$ is the fluid velocity, and $v_{p}$ is particle velocity.

To visualise the values of the parameters in the equation, the parameters of the above equation are shown in nomenclature part of the paper.

\subsection{Coupling Principle and Parameter Setting}

In this paper, Fluent-EDEM coupling simulation was adopted, where fluent 19.0 was used as the calculation tool for the fluid portion [24], and the motion of the particles within the pump was calculated by using the discrete element software EDEM 18.0. Considering the real working conditions comprehensively, the flow field and particle coupling calculations were carried out in Fluent, and the particle dynamic display was carried out in EDEM. In the iteration process, not only the dragging force, basset force, and buoyancy on each particle were calculated, but also the influence of particle shape, size, position, velocity, and other factors on the simulation flow field was obtained. Figure 3 shows the process of Fluent-EDEM coupling simulation. In the coupling process, the two-phase flow field is calculated to convergence by Fluent, and then the velocity and force information of the flow field are updated into EDEM at first. The velocity and position information of the particles is analysed by EDEM, and then the particle information is sunk into Fluent in the form of momentum for simulation. All steps are cycled until the end of the simulation.

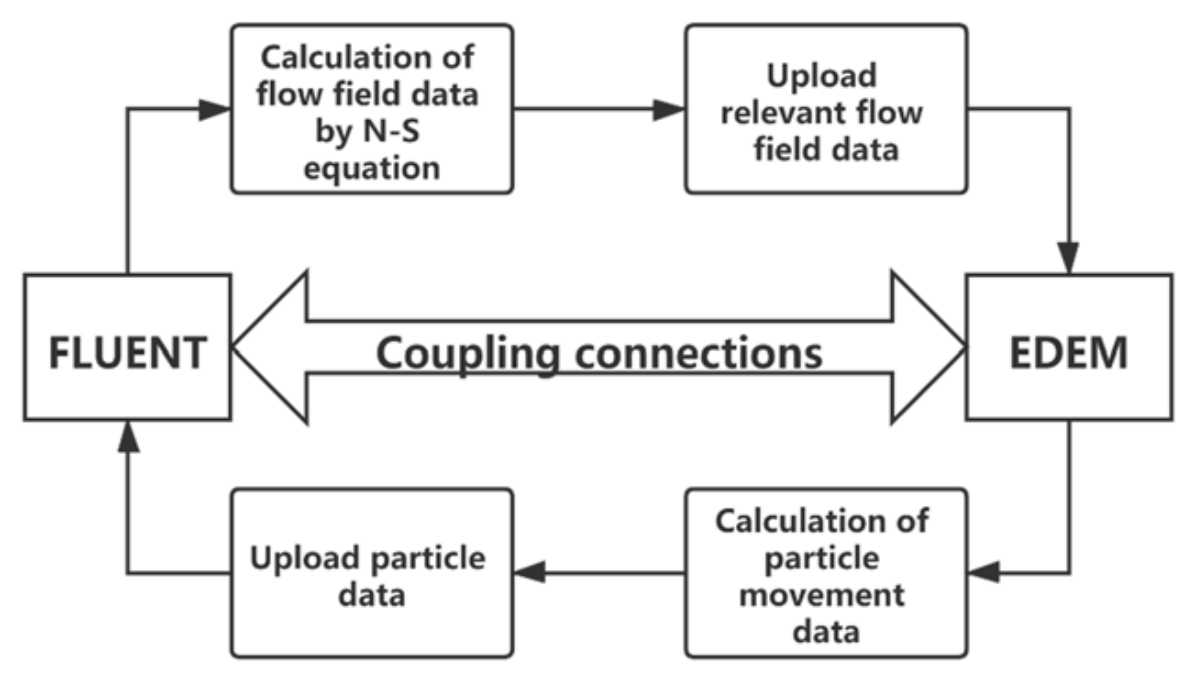

Figure 3. Schematic diagram of CFD-DEM coupling method.

Particles reflux blockage in the emergency pump stop condition is studied in this paper. After the emergency pump stop, the entire lifting system is under static pressure due to the loss of power. To simulate this state, boundary settings of velocity inlet and pressure outlet were adopted in Fluent, where the initial condition of the inlet velocity is $0 \mathrm{~m} / \mathrm{s}$. The physical properties of water in the flow field are set: the temperature is $20{ }^{\circ} \mathrm{C}$, the density is $998.2 \mathrm{~kg} / \mathrm{m}^{3}$, and the viscosity is $0.001003 \mathrm{~Pa} \cdot \mathrm{s}$. The pressure-velocity coupling method was SIMPLEC. Meanwhile, the momentum, turbulent kinetic energy, and turbulent dissipation rate have been changed to second order windward to improve the accuracy of the calculation. The choice of standard wall treatment will handle the majority of near-wall flows of $y+$ in the range of 15-100. In the coupled calculations, the data need to be transmitted in real time, and therefore, transients are adopted in the fluent calculations. In coupling, the time step in Fluent needs to be 10-100 times larger than the 
time step in EDEM. While the time step set in EDEM is $1 \times 10^{-5}$, the time step in Fluent is therefore set to $5 \times 10^{-4}$ (choose 50 times). Meanwhile, to ensure the calculation results have good engineering application value, the convergence rules for all residuals were set to $1 \times 10^{-4}$. In EDEM, the total mass of generated particles is chosen to be $50 \mathrm{~kg}$ in order to save computational resources, and the particles are selected to be generated from the start of the calculation. To study the flow capacity of the designed pump and the cause of the blockage, four particle sizes of 10-20 mm, 20-30 mm, 30-40 mm, and 40-50 $\mathrm{mm}$ were considered for simulation. The setting of particle and pump is shown in Table 2. The initial concentration of particles was set at the same concentration in EDEM, while the mass of particles generated and the simulation time was consistent with the four simulations. This simulation was for the settling of hydrostatic particles after the pump stopped. The settling velocity of the particles is shown in Table 3 [17].

Table 2. Setting of particle parameters and wall parameters.

\begin{tabular}{ccccccc}
\hline Material & $\begin{array}{c}\text { Coefficient of } \\
\text { Restitution }\end{array}$ & $\begin{array}{c}\text { Coefficient of } \\
\text { Static Friction }\end{array}$ & $\begin{array}{c}\text { Coefficient of } \\
\text { Rolling Friction }\end{array}$ & $\begin{array}{c}\text { Poisson's } \\
\text { Ratio }\end{array}$ & $\begin{array}{c}\text { Density } \\
\left(\mathbf{k g} / \mathbf{m}^{\mathbf{3}}\right)\end{array}$ & $\begin{array}{c}\text { Shear Modulus } \\
(\mathbf{M p a})\end{array}$ \\
\hline particle-particle & 0.45 & 0.28 & 0.01 & - & - & - \\
particle-pump & 0.48 & 0.16 & 0.01 & - & - & - \\
pump & - & - & - & 0.3 & 7800 & 8100 \\
particle & - & - & - & 0.4 & 2040 & 21.3 \\
\hline
\end{tabular}

Table 3. Settling velocity of the particles.

\begin{tabular}{cc}
\hline Particle $(\mathbf{m m})$ & Average Settling Velocity (m/s) \\
\hline $10-20$ & 0.613 \\
$20-30$ & 0.77 \\
$30-40$ & 0.85 \\
$40-50$ & 1 \\
\hline
\end{tabular}

\subsection{Validation}

The mesh is the link between the watershed model and the algorithmic model, so the significant position is occupied by the mesh division [10]. The choice of grid division method, quantity, and grid quality will significantly influence the calculation result. The meshing method is divided into structured meshing and unstructured meshing.

In general, the higher the mesh quality is, and the lower the mesh distortion is, the more accurate the calculation can be obtained. Li [17] has researched the clogging characteristics of single-stage pumps. The flow field when the particles reflux in the pump involves two changes: the particles enter the pump from the pipe, and the particles reflux to the pump at all stages. Therefore, the two-stage pump was chosen for the numerical simulation of particle reflux, with limited computational resources. Figure 4 shows the mesh of the two-stage centrifugal pump. In this paper, polyhedral and hexa meshes are used to divide the model.

With the RNG $k-\varepsilon$ as the calculation model, $y+$ should be best in the range of 20 to 50 . Therefore, $y+$ is chosen to be 30 . In this paper, the entire flow field in the pump is at static pressure. Only the return flow of particles causes a slight disturbance and drives the flow field. The boundary layer encryption is chosen where the flow field disturbance is large and the first grid height is $5 \times 10^{-3} \mathrm{~m}$. The purpose is to ensure the quality of the mesh on the guide vanes and impeller blades for the accuracy of the simulation calculations. 


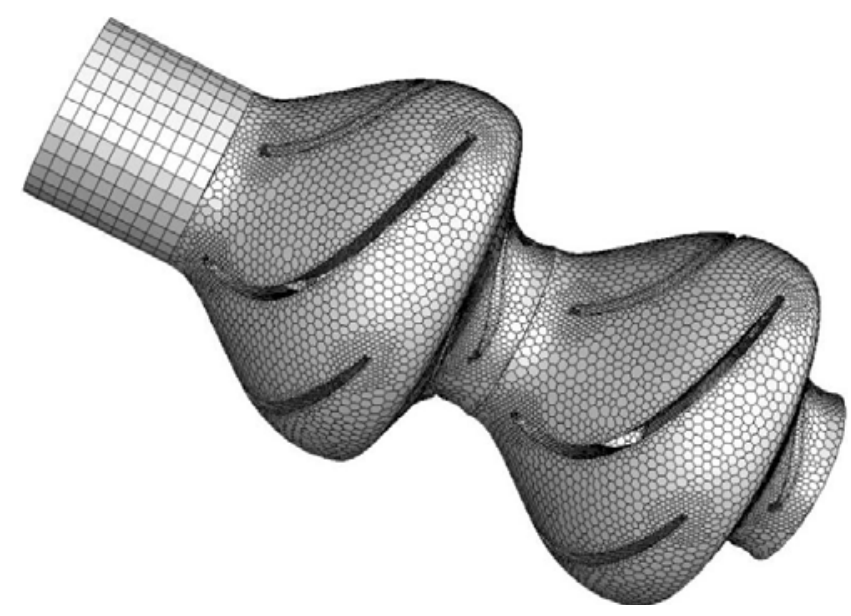

Figure 4. Mesh of two-stage centrifugal pump.

Structured and unstructured meshes of the same mesh quality can yield similar flow fields [25]. However, with different grid numbers, the settling velocity of the particle must be different in the end. The velocity comparisons obtained by grid independence verification are shown in Table 4. Determination on mesh convergence was carried out based on the relevant mesh convergence verification theory of Slater [26]. The Grid Convergence Index (GCI) reflects the superiority or inferiority of grid convergence. The corresponding values of the GCI were obtained by calculation. The safety factor $F_{s}$ was chosen to be 1.25 and after the calculation, the convergence accuracy $\mathrm{P}$ was calculated to be $1.433, G C I_{12}$ to be $0.875 \%$, and $G C I_{23}$ to be $1.18 \%$. The formula $G C I_{23} / 2^{p} G C I_{12}$ gave a calculated result of 0.99 , which is close to 1 . This indicates that the solutions are well within the asymptotic range of convergence. Given the computational speed and mesh quality, meshes of 1.14 million were chosen for the simulation. The average orthogonality of meshes was 0.8 , the average distortion of meshes was 0.21 , and the maximum distortion was only 0.615 .

Table 4. Grid independence analysis.

\begin{tabular}{ccc}
\hline Type & Grids & Average Settling Velocity of Particles $(\mathbf{m} / \mathbf{s})$ \\
\hline 1 & $3,210,564$ & 0.581 \\
2 & $1,138,252$ & 0.575 \\
3 & 274,433 & 0.559 \\
\hline
\end{tabular}

To verify the correctness of the simulation, experiments from the published paper [17] were cited for validation. The general layout of the corresponding experimental system is shown in Figure 5. The experiments were carried out in a $30 \mathrm{~m}$ high experimental system. The equipment required for the reflux experiment are water pump and water supply pipes to guarantee the hydrostatic condition, nodules silo and feeder to guarantee material supply, a pressure gauge for gauging the pressure at the inlet and outlet of the pump, and an electronic scale for gauging the weight of particles. The residual mass of $10-20 \mathrm{~mm}$ particles in the pump was measured in the reflux experiment and represented $10 \%$ of the total mass of the experimental particles. The simulation results show that the residual mass of $10-20 \mathrm{~mm}$ particles in the pump is about $1 \mathrm{~kg}$, which is $2 \%$ of the total mass of the simulated particles. The simulations were carried out with ideal particle size and sphericity, whereas the experimental particles are not perfectly spherical and may have some elliptical particles. The tolerance between simulation and test is within $10 \%$ and the tolerance is acceptable. This indicates the correctness of the simulation results. 

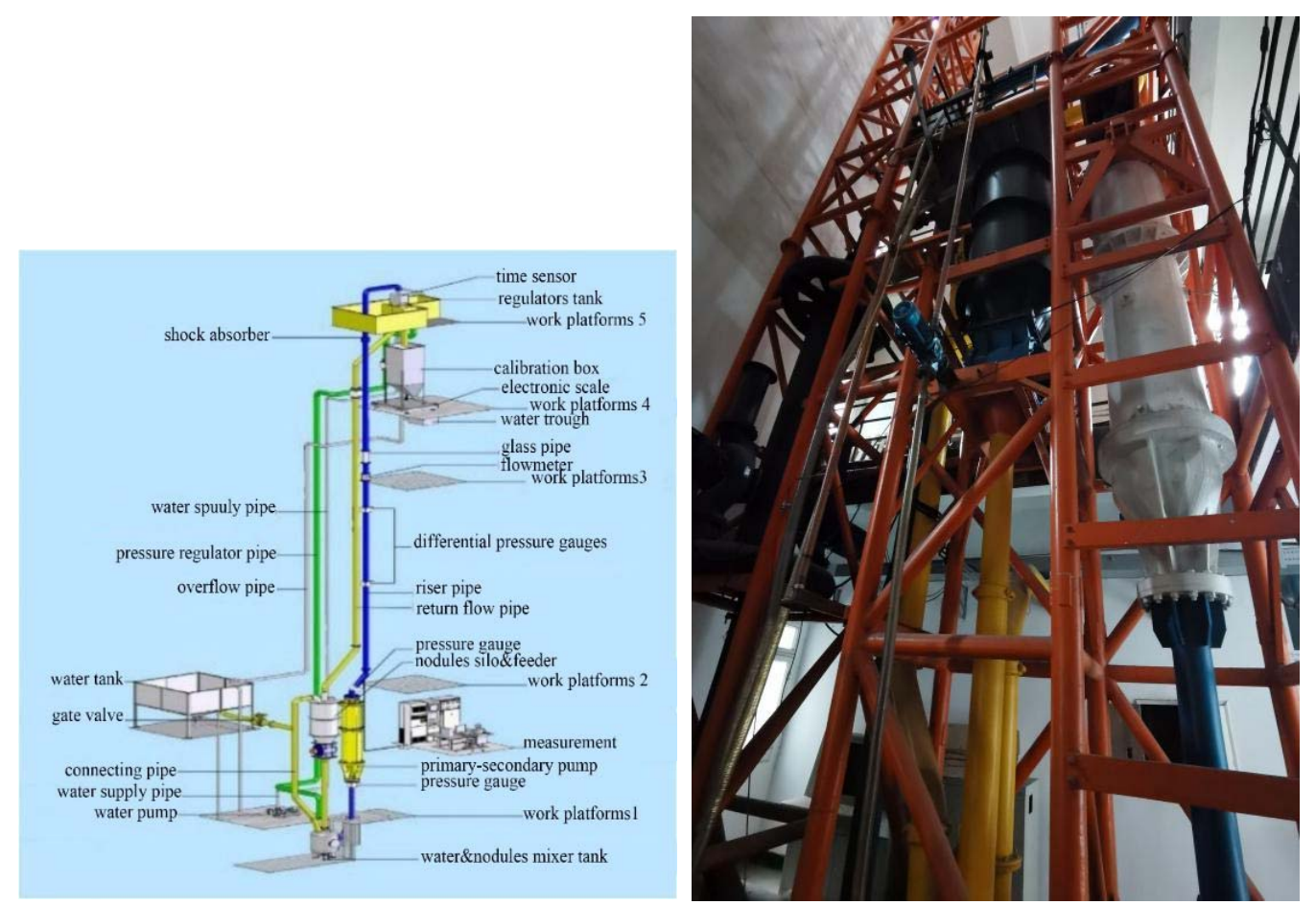

Figure 5. Layout of the experimental system.

The graph of the inlet pressure of the pump at $50 \mathrm{~mm}$ particle size is shown in Figure 6 . Comparing the two curves, it can be seen that particle blockage occurs at the inlet of the pump when refluxing at a particle size of $50 \mathrm{~mm}$, both in experiment and in simulation. The pressure on the pump inlet is sustained by the accumulation of particles pressing against the wall. The curve obtained from the simulation in Figure 6 shows a sharp drop in pressure after $1 \mathrm{~s}$, indicating that the particles in the pump are slightly relieved at this point. However, the curve gradually flattens out later, which is due to the re-accumulation of particles forming a blockage. The pressure difference between experiment and simulation corresponds to $0.025 \mathrm{Mpa}$. Considering the different contact surfaces between the particles and the pump inlet, the pressure caused on the inlet will be different and the error is within the tolerance. Hence, the corresponding pressure variation trends are consistent with the error allowance. Figure 7 is reproduced from the work of Li [17]. Furthermore, the accumulation of particles in Figure 7 shows that the simulation and the experiment are consistent, which further validates the correctness of the simulation.

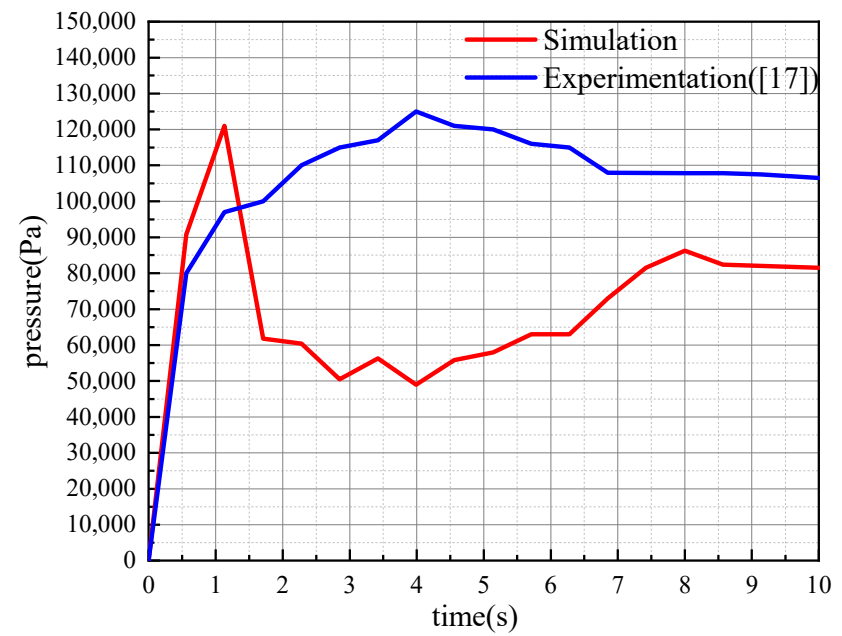

Figure 6. Graph of inlet pressure of pump at $50 \mathrm{~mm}$ particle size [17]. 


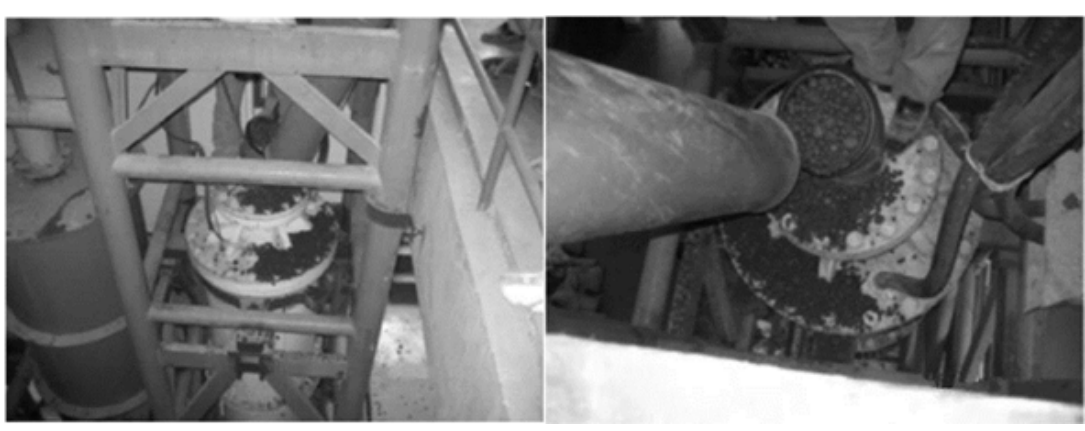

(a)

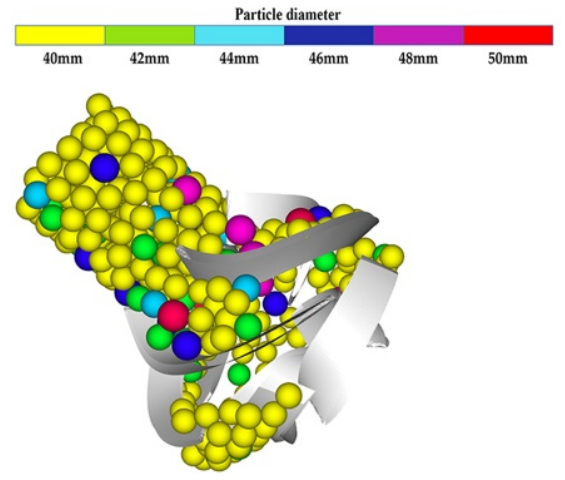

(b)

Figure 7. Particle reflux experimental and simulation comparison: (a) 40-50 mm particle reflux experimental; (b) simulation of 40-50 mm particle reflux.

\section{Simulation Results}

Four groups of mixed particles with different particle size distributions (10-20 mm, 20-30 mm, 30-40 mm, 40-50 mm) were set up in this paper, and the blockage mechanism of the four groups of mixed particles was simulated numerically. For example, the particle size interval is $2 \mathrm{~mm}$ for $10-20 \mathrm{~mm}$, which means that this group contains particles with particle sizes of $10,12,14,16,18$, and $20 \mathrm{~mm}$ at concentrations of $75 \%, 5 \%, 5 \%, 5 \%, 5 \%$, and $5 \%$, respectively. The other groups are similar.

The simulated clouds of pressure on the guide vane after collision with $10-20 \mathrm{~mm}$ particles are shown in Figure 8. It is noticeable when comparing Figure 8a,b that although both high and low-pressure areas appear on the guide vanes, the high-pressure area is denser at $10 \mathrm{~s}$ than at $2 \mathrm{~s}$. This indicates that the particles collide intensively with the guide vane at $10 \mathrm{~s}$. At the same time, these areas are found in the upper half of the guide vane close to the impeller hub and in the lower half of the guide vane away from the hub. It has been revealed that when a particle moves through a fluid, under the influence of the viscosity of the fluid, a high-pressure zone will be created ahead of the direction of motion of the particle, while a low-pressure area will be formed at the end of the trajectory and on its sides. The high-pressure and low-pressure areas on the guide vane shown in the pictures prove that the particles have good movement, and stagnation or blockage occurs. As shown in Figure 8c, except for the remaining three guide vanes, where the pressure variation zone still exists at the end of the vanes, the rest of the vanes are no longer under pressure from the particles. This demonstrates that the pre-set particles have fallen entirely, and the majority of them have passed through the interface between the guide vane and the impeller. At the same time, particles have started to flow through the impeller vanes to the next stage of the pump.

However, when studying the pressure variation of 20-30 $\mathrm{mm}$ particles on the guide vane of the first stage pump, the pressure zone distribution of particles on the vane was essentially the same as for 10-20 mm particles. The pressure clouds are shown in Figure 9, and from Figure $9 b$, it can be seen that the high-pressure points on the guide vane are much denser for 20-30 $\mathrm{mm}$ particles than for 10-20 $\mathrm{mm}$ particles. Those reflect that the collision of 20-30 mm particles in the guide vane channel is much larger than in the previous particle class. Meanwhile, the denser movement of particles in the flow channel means an increased risk of multiple particles squeezing each other, which increases the risk of blockages. 


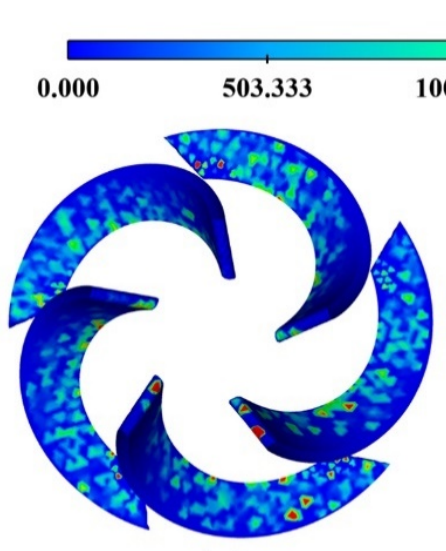

(a)

Pressure(Pa)

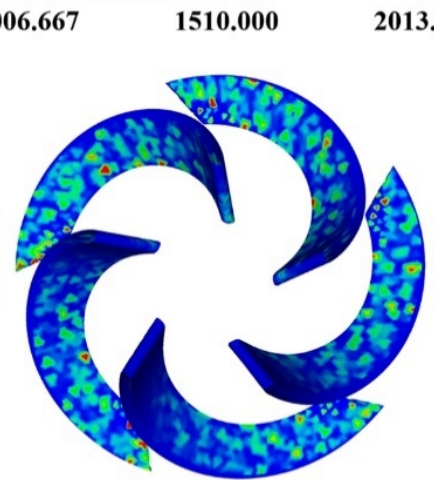

(b)

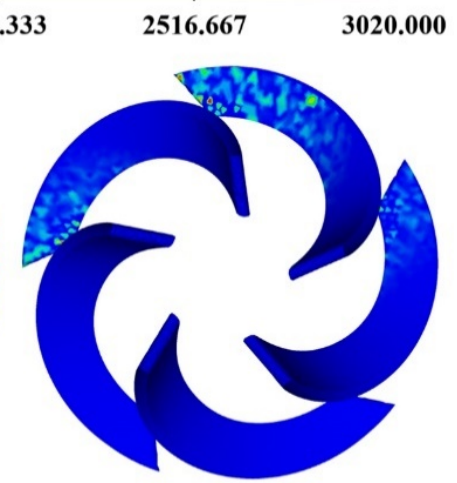

(c)

Figure 8. Pressure cloud on the guide vane after being collided with 10-20 mm particles: (a) pressure cloud graph at $2 \mathrm{~s}$; (b) pressure cloud graph at10 s; (c) pressure cloud graph at $15 \mathrm{~s}$.

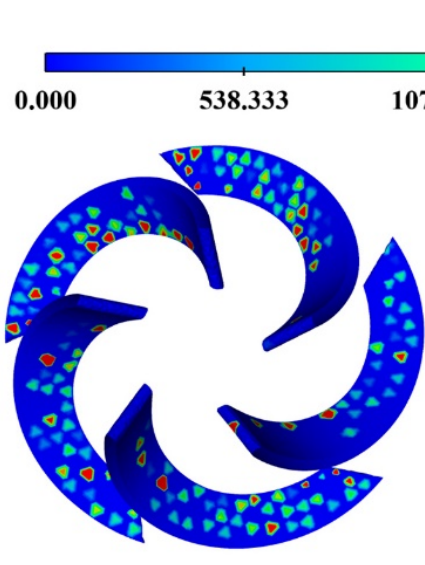

(a)

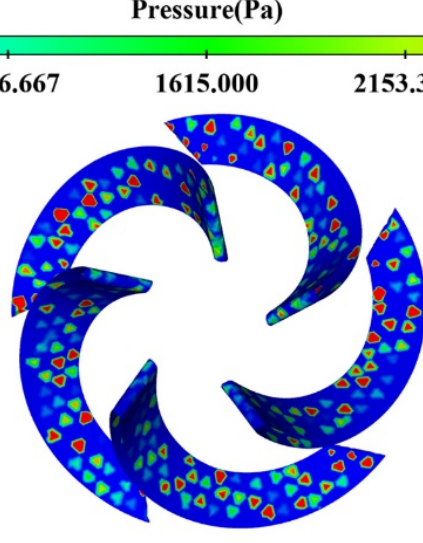

(b)

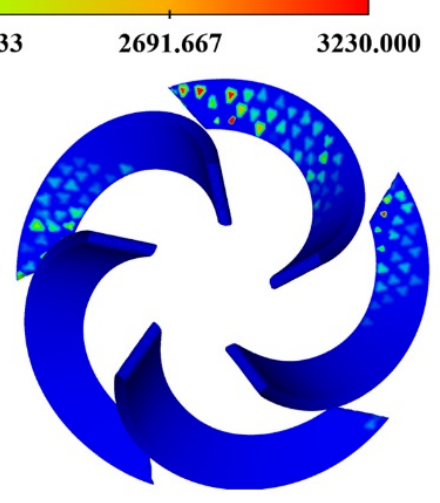

(c)

Figure 9. Pressure cloud on the guide vane after being collided with 20-30 mm particles: (a) pressure cloud graph at $2 \mathrm{~s}$; (b) pressure cloud graph at $10 \mathrm{~s}$; (c) pressure cloud graph at $15 \mathrm{~s}$.

Comparisons between Figures 8 and 9 reveal that the behaviour of the $10-20 \mathrm{~mm}$ and 20-30 mm particles was basically the same: at $2 \mathrm{~s}$, the particles fall without hindrance. The particles exert pressure mainly on the middle and the lower half of the guide vanes. However, at $10 \mathrm{~s}$, temporary accumulation of particles in the pump occurs because the previous particles cannot fall into the next pump stage in time. This leads to the phenomenon of pressure areas spreading all over the guide vane. At $15 \mathrm{~s}$, the particles have almost finished falling. However, residual particles remain in some of the flow channels, which leads to the phenomenon of pressure zones still existing on some of the guide vanes.

The movement and accumulation of 10-20 and 20-30 mm particles at the corresponding time points are illustrated in Figure 10. The behaviour of two particle sizes in the pump is compared to reveal the clogging mechanism of the particles better. From Figure 10a,c, it can be analysed that there is a velocity gradation in the particles that accumulate in the guide vane of the first stage pump. Figure 10a shows that the particle velocity in this region ranges from $0.019 \mathrm{~m} / \mathrm{s}$ to $0.165 \mathrm{~m} / \mathrm{s}$, while in Figure 10c, it ranges from $0 \mathrm{~m} / \mathrm{s}$ to $0.213 \mathrm{~m} / \mathrm{s}$. This indicates that the particles are not jammed in the flow channels. The static particles are mainly concentrated at the interface between the guide vane and the impeller. This is the temporary accumulation of particles caused by the front particles not falling in time. Furthermore, at the interface between the primary pump and the two stages, the particles can be seen falling at a speed in the range of $0.4-0.8 \mathrm{~m} / \mathrm{s}$. This indicates that the particles 
are not blocked in the first stage pump. Hence, it can be seen that $10-30 \mathrm{~mm}$ particles have good movement characteristics in the pump. Comparison of Figure 10b,d shows that the reflux completion time for $20-30 \mathrm{~mm}$ particles is shorter than $10-20 \mathrm{~mm}$ particles. This suggests that larger particles can be better returned to the flow with the assurance of non-clogging. This is because of the restricted particle movement caused by the collision between many particles, which eventually leads to a decrease in the reflux velocity.

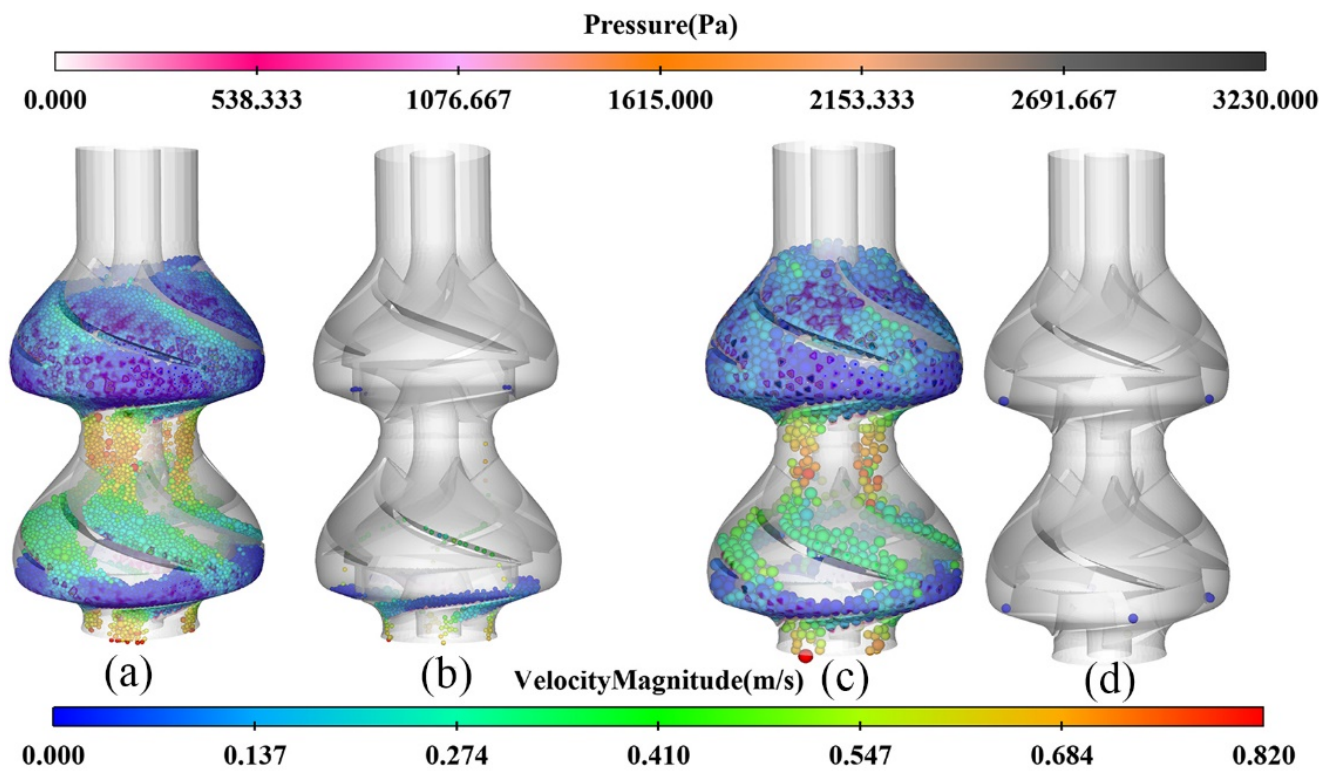

Figure 10. The movement and accumulation of 10-20 and 20-30 $\mathrm{mm}$ particles at the corresponding time points: (a) 10-20 mm particles at $10 \mathrm{~s}$ simulation; (b) 10-20 $\mathrm{mm}$ particles at $30 \mathrm{~s}$ simulation; (c) 20-30 mm particles at $10 \mathrm{~s}$ simulation; (d) 20-30 mm particles at $30 \mathrm{~s}$ simulation.

However, a more noticeable variation in particle reflux can be found in the study of Figure 11 for 30-40 mm than for the previous particle classes. As can be seen in Figure 11a, different pressure distribution areas are formed on the guide vanes because of particle collisions and rolling during the reflux process. In comparison with Figures 8 and 11a, it is found that the pressure distribution area of one of the guide vanes is not continuous, and the lower half of the guide vanes is not under pressure from the particles in Figure 11a. In our analysis, we established that the two sides of a guide vane channel are made up of the guide vane blades' inner concave and outer convex surfaces. The high-pressure point is also found in the corresponding outer convex surface of the guide vane in the guide vane flow channel, where the pressure point is discontinuous compared to Figure 11b. Therefore, it can be tentatively concluded that the particles are probably blocked in this channel. The high-pressure points on both sides of the flow channel reflect how the particles are clogged by the stacking of multiple particles, causing jamming. Further support for the previous speculation is provided by comparing the distribution of high-pressure points in Figure $11 \mathrm{c}, \mathrm{d}$. 


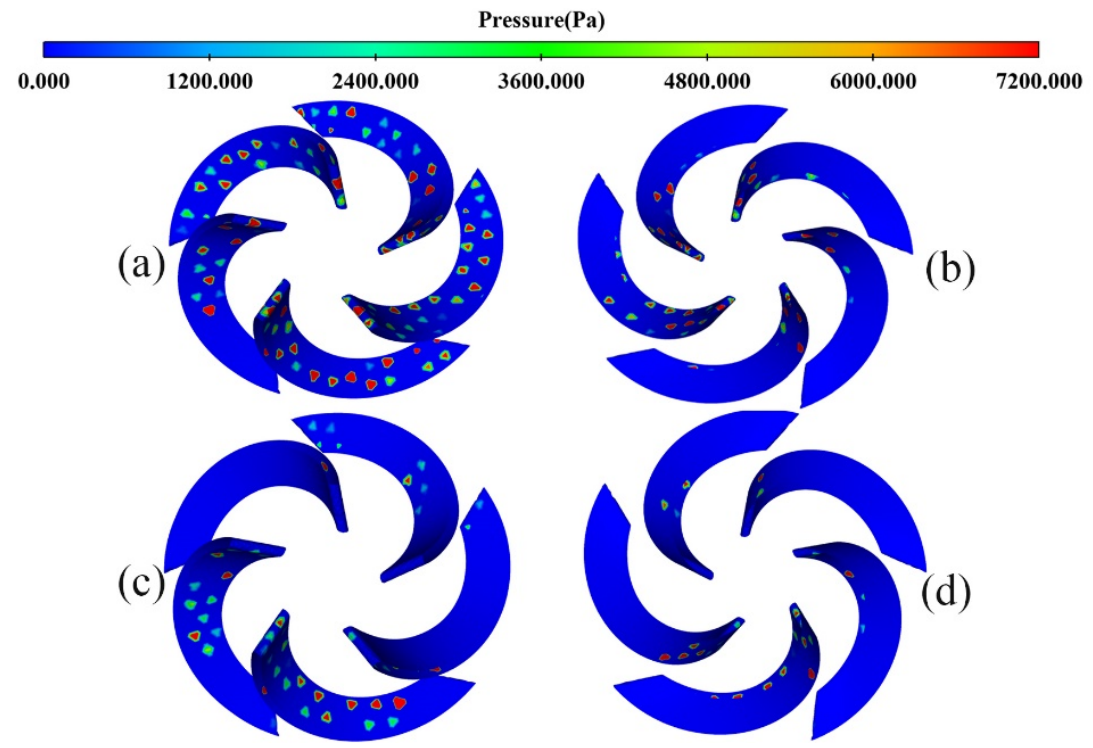

Figure 11. Pressure cloud on the guide vane after being collided with $30-40 \mathrm{~mm}$ particles: (a) simulation of the pressure on the inner concave surface of the guide vane at $10 \mathrm{~s}$; (b) simulation of forces on the outer convex surface of the guide vane at $10 \mathrm{~s}$; (c) simulation of the pressure on the inner concave surface of the guide vane at $30 \mathrm{~s}$; (d) simulation of the pressure on the inner convex surface of the guide vane at $30 \mathrm{~s}$.

The movement of $30-40 \mathrm{~mm}$ particles in the pump is illustrated in Figure 12 . As shown in Figure 12a, one of the flow channels in the first stage pump has been blocked. Figure $12 \mathrm{~b}$ shows the blocked section where particles remain in the first stage pump. The high-pressure area of the pump body can be analysed to reveal the blockage caused by the stacking of multiple particles, which more visually confirms the previous suspicions.

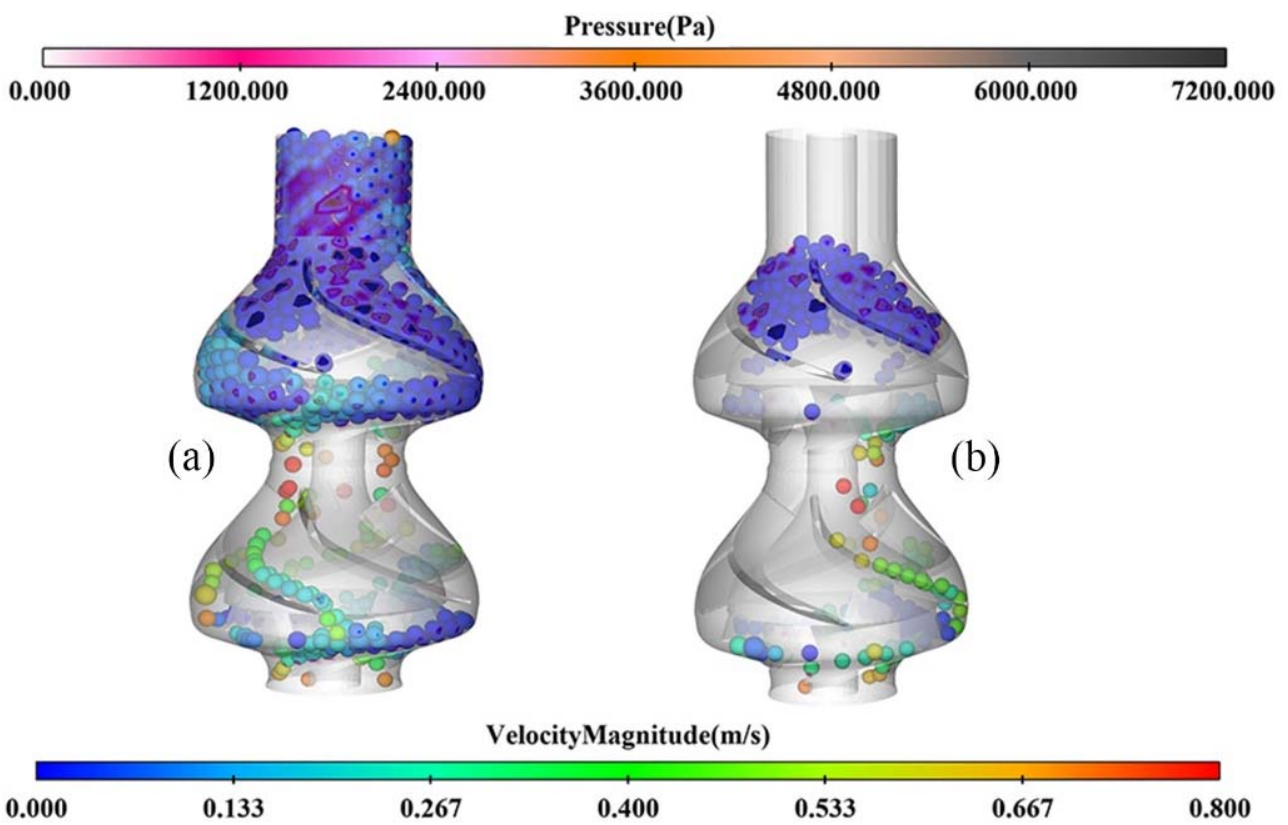

Figure 12. Movement and accumulation of 30-40 $\mathrm{mm}$ particles in the pump: (a) 30-40 mm particles at $10 \mathrm{~s}$ simulation; (b) $30-40 \mathrm{~mm}$ particles at $30 \mathrm{~s}$ simulation.

Research on the reflux of $40-50 \mathrm{~mm}$ particles in the pump revealed that the pressure distribution in the guide vane is similar to that of $30-40 \mathrm{~mm}$ particles, while the flow of particles in the pump is further changed compared to $30-40 \mathrm{~mm}$ particles. As shown in 
Figure 13, the blockages are more severe at this particle size. Figure 13a demonstrates the particle reflux when the simulation proceeds to $5 \mathrm{~s}$. The diagram shows that particles have blocked two of the flow channels in the first stage of the pump, while the remaining flow channels have the majority of the particle flow velocity at $0.007 \mathrm{~m} / \mathrm{s}$. Figure $13 \mathrm{~b}$ shows the simulation as it proceeds to $10 \mathrm{~s}$. As seen from Figure 13b, the particles are completely blocked in the first stage of the pump, and all flow channels are blocked.

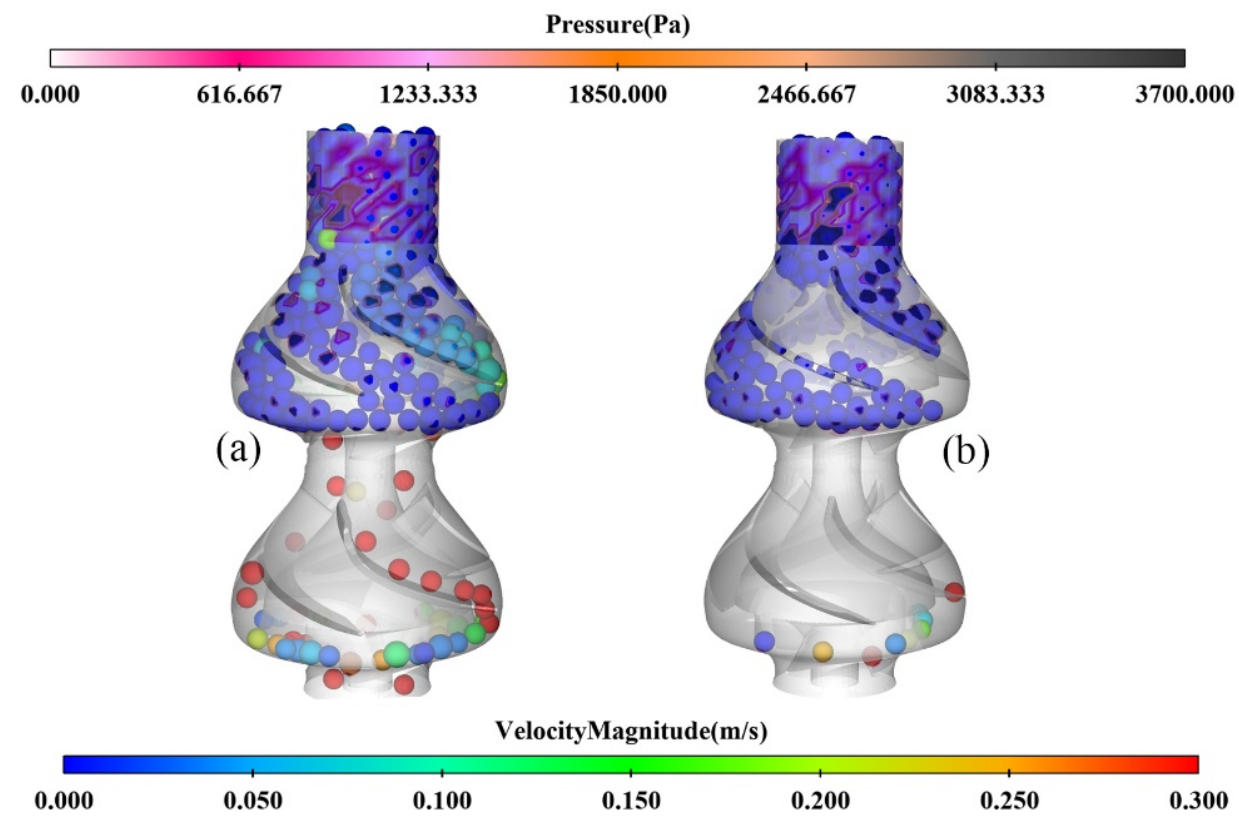

Figure 13. Movement and accumulation of 40-50 mm particles in the pump: (a) 40-50 mm particles at $5 \mathrm{~s}$ simulation; (b) $40-50 \mathrm{~mm}$ particles at $15 \mathrm{~s}$ simulation.

Once blockages occur in the pump, the accumulated particles will squeeze the flow channels. This will exert pressure on the components in the channels. The worse the blockage, the greater the pressure on the corresponding component. Therefore, the pressure on the components can also reflect the degree of blockage of particles in the pump. The graph in Figure 14 shows the maximum pressure variation trend for different particle sizes on the first stage guide vane of the pump. The fluctuations of the four curves in Figure 14 represent that the particles are capable of moving in the flow channel during that period. While when pressure is stabilised the severity of the blockage is reflected by the pressure level. As shown in Figure 14a,b, the pressure on the guide vane for both 10-20 and 20-30 mm particles becomes 0 Pa over time. Meanwhile, the pressure of 20-30 mm particles on the guide vane is significantly higher than that of $10-20 \mathrm{~mm}$ particles, which can be observed from the graph. Therefore, it can be deduced that the collision level of $20-30 \mathrm{~mm}$ particles in the guide vane flow channel is stronger than that of $10-20 \mathrm{~mm}$ particles.

On the other hand, the pressure of $30-40 \mathrm{~mm}$ particles on the guide vane is stable at $0.3 \mathrm{MPa}$ when the value ceases to vary, as shown in Figure 14c. Therefore, a blockage of the particles in the pump can be determined at this particle size. However, $40-50 \mathrm{~mm}$ particles were found to block quickly in the pump, as shown in Figure 14d. The pressure of the particles on the guide vane is maintained at 1.4 MPa. This suggests that the particles are rapidly and severely blocked in the pump. 


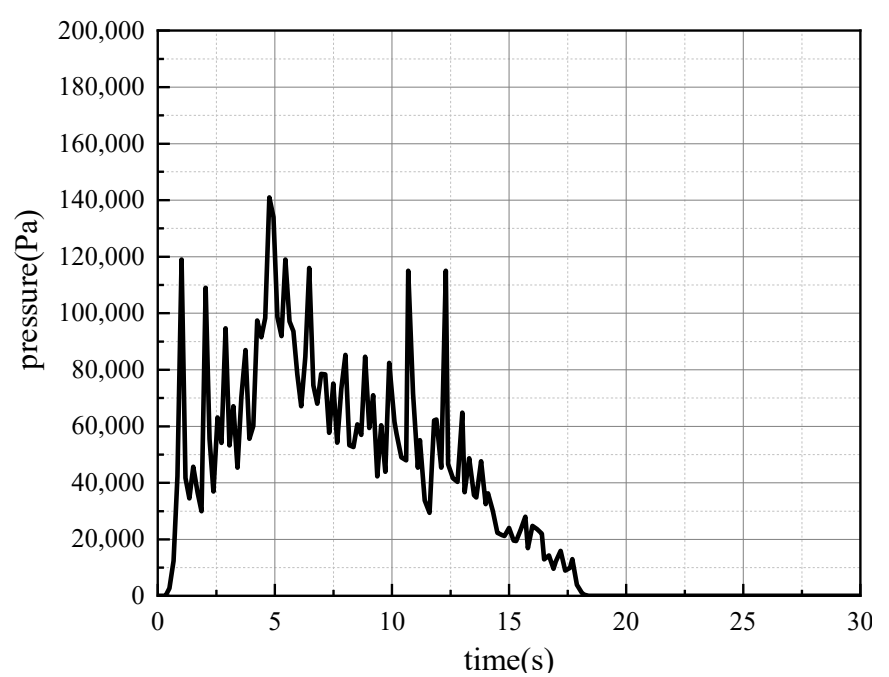

(a)

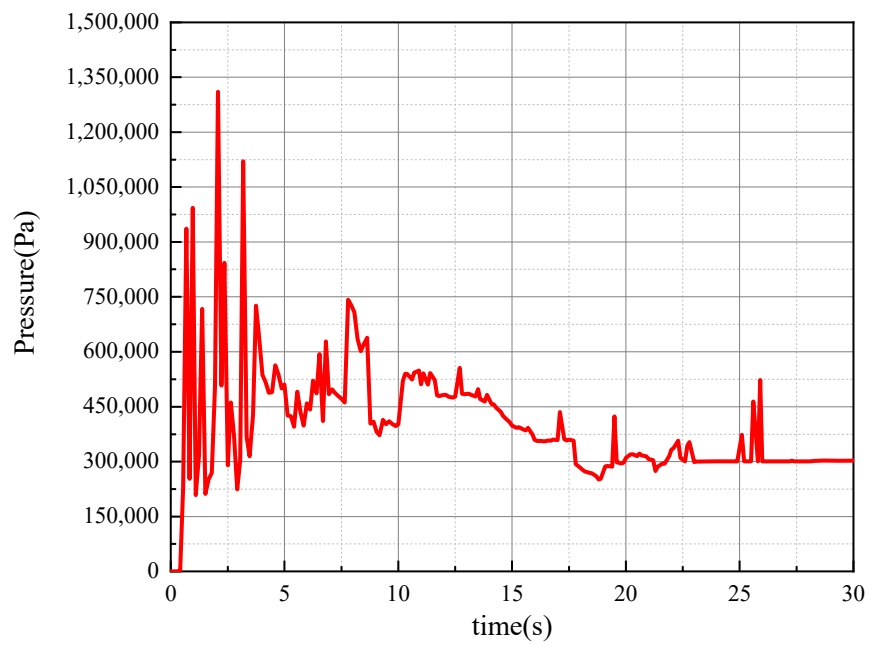

(c)

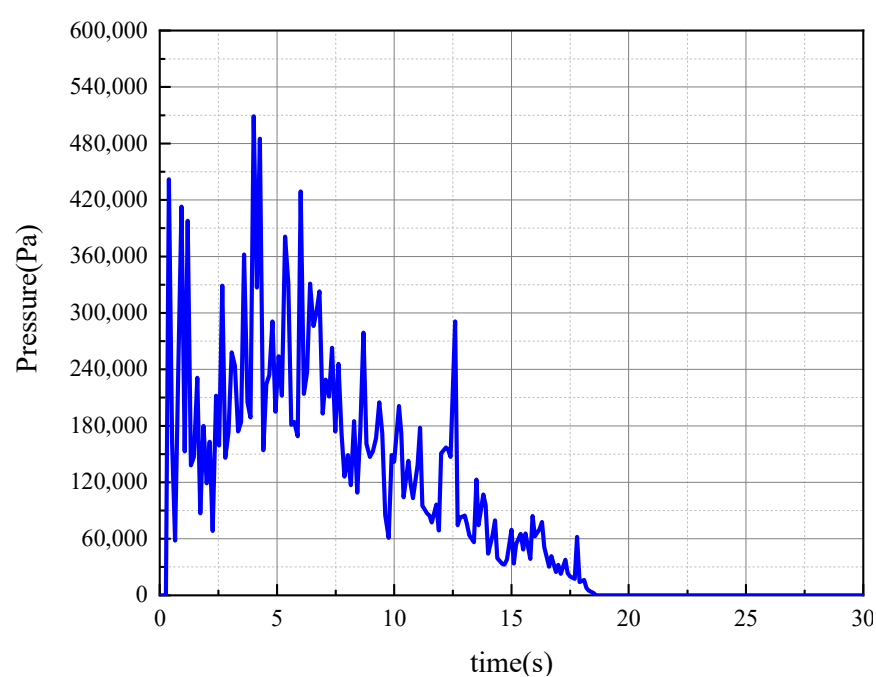

(b)

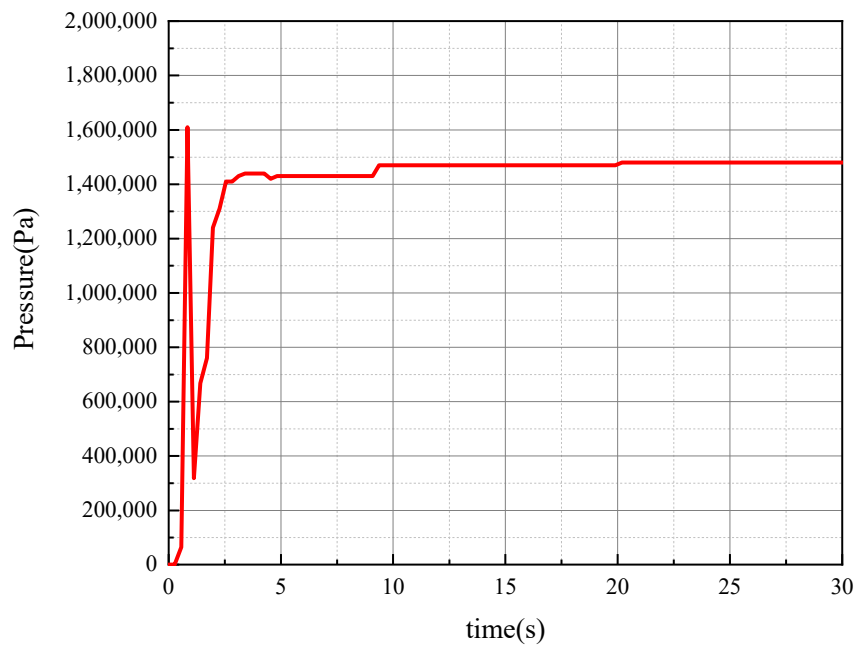

(d)

Figure 14. Maximum pressure variation on the guide vane of the first stage pump with different particle sizes: (a) 10-20 mm; (b) 20-30 mm; (c) 30-40 mm; (d) 40-50 mm.

Figure 15 displays the tendency of the particle mass in the pump. As can be seen from the graphs, the total mass of 10-20 and 20-30 mm particles in the pump have almost the same tendency to change, and the particles are entirely refluxed. Simultaneously, the reflux velocity of $20-30 \mathrm{~mm}$ particles is slightly faster than that of $10-20 \mathrm{~mm}$ particles. The reflux velocity of $30-40 \mathrm{~mm}$ particles is significantly lower than that of the previous particle sizes, and some of the particles ultimately remain partially in the pump. There are some fluctuations in the peak part of each of the four curves, which results from the fact that the reflux velocity varies for different particle sizes. When some of the particles leave the pump from the outlet while particles are still being generated at the inlet of the pump, it can cause the curve to fluctuate. The reason why the four curves intersect at different points in time is that particles of different sizes have different levels of blockage in the pump. When particles block in the pump, it can slow down the reflux of particles in the pump. Situations such as 30-40 mm particles' mass curves intersecting other curves can occur. In combination with Figures 10,12 and 13, it is caused by particles forming blocked sections in the first stage pump, where some particles are unable to complete the reflux. The mass profile of $40-50 \mathrm{~mm}$ particles reflects the fact that the particles are heavily blocked in the pump and the particles are unable to complete the reflux. 


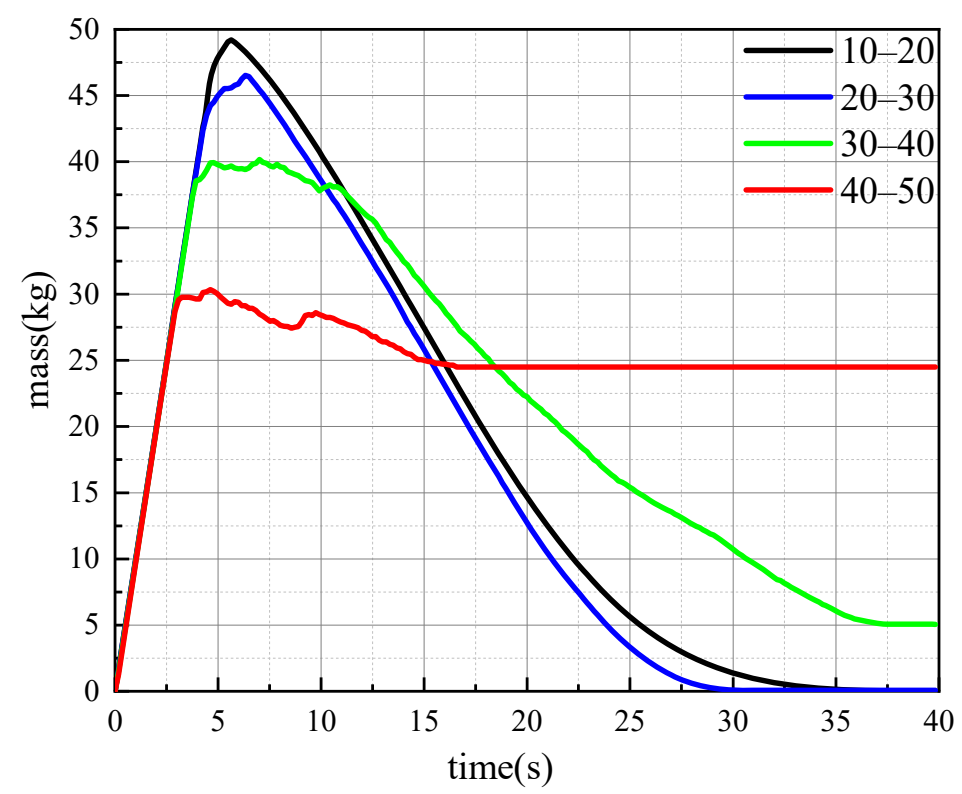

Figure 15. Particle mass in the pump.

The effect of particle size on particle reflux can be tentatively concluded from the above simulation results. As the proportion of particle size distribution is consistent across the four groups of simulations, the effect of particle size distribution on reflux needs to be studied more directly. However, the blockage was more noticeable at $40-50 \mathrm{~mm}$ than at other particle sizes, and two additional groups of control trials were considered in this particle size range: simulation of the single-particle size of $40 \mathrm{~mm}$ and the particle size distribution of $40 \mathrm{~mm}$ ( $85 \%$ concentration), $42 \mathrm{~mm}$ ( $5 \%$ concentration), $44 \mathrm{~mm}$ (5\% concentration), $50 \mathrm{~mm}$ ( $5 \%$ concentration).

As shown in Figure 16, it is the different particle size distributions that lead to blockages with different degrees. In addition, the particle size is differentiated by colour for more accessible research on particle blockage behaviour: $40 \mathrm{~mm}$-yellow, $42 \mathrm{~mm}$-green, $44 \mathrm{~mm}$-sky blue, $46 \mathrm{~mm}$-blue, $48 \mathrm{~mm}$ - purple, $50 \mathrm{~mm}$-red. As shown in Figure 16a, the blockage of $40 \mathrm{~mm}$ particles in the guide vane flow channel is mainly caused by several particles squeezing each other in the flow channel and finally forming a triangular nodular blockage. Particles of different particle sizes can easily stack with each other at the interfaces of the guide vanes and impellers, and the phenomenon can be seen in Figure 16b. This phenomenon leads to large particles forming nodular blockages, while smaller particles following closely behind will become stuck in the gaps between the larger particles, creating even more severe blockages. Although blockage occurs in Figure 16c, d, it is relatively slight compared to Figure 16b. Combined with the curves in Figures 15 and 17 again, it can be seen that the selection of larger particles is within the permissible range of particle size distribution. This has a certain improvement in terms of the passage of particles through the pump in the reflux. Meanwhile, the principles that caused the fluctuations and intersections of the curves in Figure 17 were the same as those in Figure 15.

The line graphs that count the particle mass remaining in the first stage pump for the four situations of particle blockage section are shown in Figure 17. Comparing the particle size distributions of $40-42-44-50 \mathrm{~mm}, 40-46-48-50 \mathrm{~mm}$, and $40-50 \mathrm{~mm}$, it is found that particles with the particle size distribution of $40-42-44-50 \mathrm{~mm}$ are the most serious in the pump blockage situation. The blockage of particles with size distributions of 40-46$48-50 \mathrm{~mm}$ is less severe than that of particles with size distributions of $40-50 \mathrm{~mm}$. While the blocking of single-particle size in the pump is the lightest, it can be concluded that when the particle size exceeds the threshold for blockage, large particles can cause severe blockage in the pump. In terms of particle size distribution, larger particles in a given particle size distribution can effectively improve the reflux of particles. 


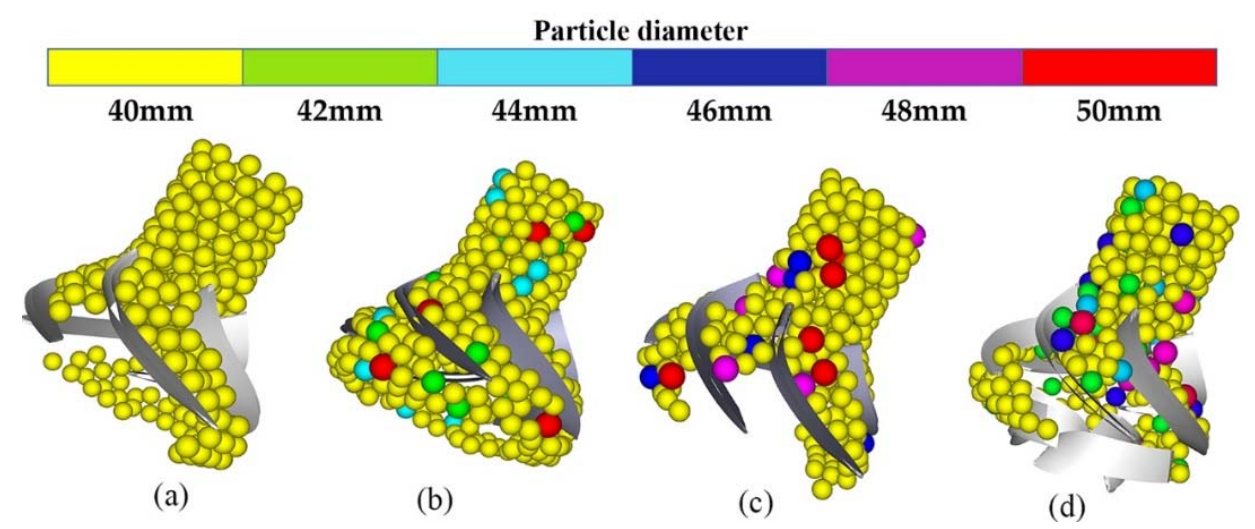

Figure 16. Different particle size distributions that lead to blockages with different degrees: (a) single-particle size of $40 \mathrm{~mm}$; (b) particle size distribution of 40,42, 44, and $50 \mathrm{~mm}$; (c) particle size distribution of 40, 46, 48, and $50 \mathrm{~mm}$; (d) particle size distribution of 40,42,44, 46, 48, and $50 \mathrm{~mm}$.

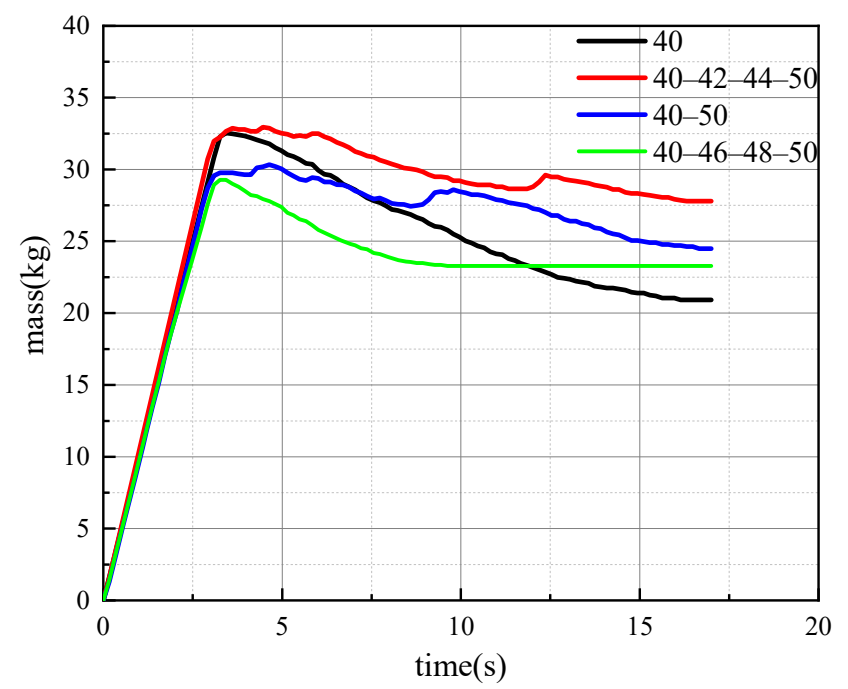

Figure 17. Blockage mass of particles with different particle size distributions in the first pump stage.

\section{Conclusions}

A numerical simulation was carried out for the backflow problem of an emergency shutdown of a multistage centrifugal pump. The following conclusions were drawn.

First, the concentration of particles has a specific influence on the backflow. When the falling velocity of particles is faster than the velocity of particles leaving the lower cover plate of the impeller, the concentration of the particles can reach a very high level locally. Then, the extrusion pressure of particles will make particles agglomerate, causing blockage.

Second, the backflow can be completed well in a size of $10-30 \mathrm{~mm}$. At the same time, 30-40 mm particles will form part of the blocked section remaining in the pump with the primary particles of $30 \mathrm{~mm}$. The analysis reveals that particles of $40-50 \mathrm{~mm}$ can cause severe blockages. The maximum diameter of particles should be controlled within $30 \mathrm{~mm}$ to ensure ore yield and equipment safety.

Third, the simulation shows that the interface between the guide vane and the impeller is more prone to forming an accumulation of particles. The squeezing motion of multiple particles against each other can easily lead to the blockage of segments.

Fourth, research into the movement behaviour of particles has revealed that particle blockage is caused by multiple large particles placed in one section of the flow channel at the same time, which restrict the movement between the particles and eventually block into the flow channel. At the same time, smaller particles at the back tend to fill in the gaps between the larger particles as they form blockage segments. This eventually leads to 
severe blockages occurring. Therefore, the size distribution of the particles significantly influences the reflux of the particles. It is recommended to use coarse particles with a larger particle size to ensure a safe return flow in the pump under the premise of no blockages.

Author Contributions: Conceptualization, J.C. and Q.H.; Methodology, J.C.; Software, L.D. and J.C.; Validation, Q.H., Y.K. and S.L.; Formal analysis, J.C.; Investigation, J.C., L.D. and Y.K.; Resources, Q.H. and S.L.; Data curation, J.C.; Writing—original draft preparation, J.C.; Writing—review and editing, J.C., L.D. and Q.H.; Visualization, J.C.; Supervision, Q.H. and S.L.; Project administration, S.L.; Funding acquisition, S.L. All authors have read and agreed to the published version of the manuscript.

Funding: This project was supported by the National Key Research and Development Project of China (2016YFC0304103), National Key Research and Development Program of China (2019YFC0312405), Open-end Fund of State Key Laboratory of Deep-sea Mineral Resources Development and Utilization Technology (SH-2020-KF-A02), Natural Science Foundation of Hunan Province (2021JJ30824) Experimental Study on Solid-liquid Two-Phase Flow of Coarse Particles in Deep-sea Electric lifting Pump.

Institutional Review Board Statement: Not applicable.

Informed Consent Statement: Not applicable.

Data Availability Statement: Not applicable.

Acknowledgments: The authors would like to thank the anonymous reviewers for their constructive suggestions which comprehensively improved the quality of the paper.

Conflicts of Interest: The authors declare no conflict of interest.

\section{Nomenclature}

$C_{D} \quad$ coefficient of drag force

d particle diameter

$E^{*} \quad$ Young's modulus

$F_{D} \quad$ fluid drag force

$F_{f p, i} \quad$ interaction forces between continuous and discrete phases

$F_{M} \quad$ lift force generated by the rotation of particles in the flow field

$F_{S} \quad$ lift force due to fluid shear

$F_{n, i j}, F_{t, i j} \quad$ normal and tangential contact force between particles $i$ and $j$

$G_{b}, G_{k} \quad$ turbulence kinetic energy

$G^{*} \quad$ Shear modulus

$I_{i} \quad$ moment of the particle

$k \quad$ turbulent momentum

$R \quad$ diameter of particles

$S_{k}, S_{\varepsilon} \quad$ source terms

$M_{i} \quad$ mass

$M_{t, i j}, M_{r, i j} \quad$ tangential and rolling frictional torques

$u_{f} \quad$ fluid velocity

$v_{i} \quad$ translational velocity

$v_{t}^{\text {rel }} \quad$ tangential component

$w_{i} \quad$ rotational velocity

$x_{i}, x_{j} \quad$ direction

$Y_{M} \quad$ dissipation rate

$\mu_{f} \quad$ liquid's viscosity

$\varepsilon \quad$ turbulent dissipation rate

$\rho$ density of the liquid

$u_{i} \quad$ direction of liquid flow

$\alpha$ normal overlap distance 


\section{References}

1. Hu, Q.; Zou, L.; Lv, T.; Guan, Y.J.; Sun, T.Z. Experimental and Numerical Investigation on the Transport Characteristics of Particle-Fluid Mixture in Y-Shaped Elbow. J. Mar. Sci. Eng. 2020, 8, 675. [CrossRef]

2. Kang, Y.J.; Liu, S.J.; Hu, X.Z.; Dai, Y. Numerical and Experimental Investigation of a Multistage Electric Lifting Pump for Deep-Sea Mining. In Proceedings of the 2018 3rd International Conference on Smart City and Systems Engineering (ICSCSE), Xiamen, China, 29-30 December 2018; pp. 457-462.

3. Dai, Y.; Xue, C.; Su, Q. An Integrated Dynamic Model and Optimized Fuzzy Controller for Path Tracking of Deep-Sea Mining Vehicle. J. Mar. Sci. Eng. 2021, 9, 249. [CrossRef]

4. Zou, W.S.; Lu, Y.; Li, Z. Numerical simulation analysis of deep-sea mining lift pump. J. Hunan Univ. (Nat. Sci. Ed.) 2013, 40, 59-63.

5. Yang, H.L.; Liu, S.J.; Zou, W.S. Research on deep-sea mining high head coarse particles multistage transfer electric pump. J. Cent. South Univ. (Nat. Sci. Ed.) 2018, 49, 1110-1117.

6. Plua, F.; Hidalgo, V.; López-Jiménez, P.A.; Pérez-Sánchez, M. Analysis of Applicability of CFD Numerical Studies Applied to Problem When Pump Working as Turbine. Water 2021, 13, 2134. [CrossRef]

7. Huang, B.; Zeng, G.; Qian, B.; Wu, P.; Shi, P.; Qian, D. Pressure Fluctuation Reduction of a Centrifugal Pump by Blade Trailing Edge Modification. Processes 2021, 9, 1408. [CrossRef]

8. Zeng, Y.; Chen, Q.; Xie, Q.; Li, F. Analysis of the Effect of Particle Diameter on Solid-liquid Two-phase Flow in a Lifting Pump of Deep Sea Mining. J. Xuzhou Inst. Technol. (Nat. Sci. Ed.) 2013, 28, 46-52.

9. Dong, Z.; Liu, S.J.; Hu, X.Z.; Wen, H.; Ge, P. Research on the calculation method of two-phase flow of coarse particles. Mar. Eng. 2018, 36, 110-116.

10. Cundall, P.A.; Strack, O.D. A discrete numerical model for granular assemblies. Geotechnique 1979, 29, 47-65. [CrossRef]

11. Zhang, A.; Jiang, M.; Thornton, C. A coupled CFD-DEM method with moving mesh for simulating undrained triaxial tests on granular soils. Granul. Matter 2020, 22, 13. [CrossRef]

12. Yao, L.; Xiao, Z.; Liu, J.; Zhang, Q.; Wang, M. An optimized CFD-DEM method for fluid-particle coupling dynamics analysis. Int J. Mech. Sci. 2020, 174, 105503. [CrossRef]

13. Razavi, F.; Komrakova, A.; Lange, C.F. CFD-DEM Simulation of Sand-Retention Mechanisms in Slurry Flow. Energies 2021, 14, 3797. [CrossRef]

14. Tang, Z.; Yu, L.; Wang, F.; Li, N.; Chang, L.; Cui, N. Effect of Particle Size and Shape on Separation in a Hydrocyclone. Water 2019, 11, 16. [CrossRef]

15. Li, Y. Solid-Liquid Two-Phase CFD-DEM Method in Variable Curvature Channel and Its Application in Large-Scale Desulfurization Pump; Jiangsu University: Zhenjian, China, 2015.

16. Deng, L.; Hu, Q.; Chen, J.; Kang, Y.; Liu, S. Particle Distribution and Motion in Six-Stage Centrifugal Pump by Means of Slurry Experiment and CFD-DEM Simulation. J. Mar. Sci. Eng. 2021, 9, 716. [CrossRef]

17. Li, Y.W.; Liu, S.J.; Hu, X.Z. Research on reflux in deep-sea mining pump based on DEM-CFD. Mar. Georesour. Geotechnol. 2020, 38, 744-752.

18. Kang, Y.J.; Liu, S.J.; Zou, W.S.; Zhao, H.; Hu, X. Design and analysis of an innovative deep-sea lifting motor pump. Appl. Ocean. Res. 2019, 82, 22-31. [CrossRef]

19. Kang, Y.J.; Liu, S.J.; Zou, W.S.; Hu, X.; Manzoni, S. Numerical Investigation on Pressure Pulsation Characteristics and Radial Force of a Deep-Sea Electric Lifting Pump at Off-Design Conditions. Shock Vib. 2019, 2019, 4707039. [CrossRef]

20. Zhang, S.; Li, X.; Zhu, B.; Hu, Q. Applicability of k- $\varepsilon$ Eddy Viscosity Turbulence Models on Numerical Simulation of Centrifugal Pump. J. Mech. Eng. 2009, 45, 238-242. [CrossRef]

21. Yakhot, V.; Orszag, S.A. Renormalization group analysis of turbulence. I. Basic theory. J. Sci. Comput. 1986, 1, 3-51. [CrossRef]

22. Chen, J.; Shi, W.; Zhang, D. Influence of blade inlet angle on the performance of a single blade centrifugal pump. Eng. Appl. Comp. Fluid Mech. 2021, 15, 462-475.

23. Su, X.H.; Tang, Z.; Li, Y.; Zhu, Z.; Mianowicz, K.; Balaz, P. Research of Particle Motion in a Two-Stage Slurry Transport Pump for Deep-Ocean Mining by the CFD-DEM Method. Energies 2020, 13, 6711. [CrossRef]

24. ANSYS Inc. ANSYS Fluent 19.0 User's Guide; ANSYS Inc.: Canonsburg, PA, USA, 2019; Volume 1, pp. 39-127.

25. Liu, H.L.; Liu, M.M.; Bai, Y.; Liang, D. Effects of mesh style and grid convergence on numerical simulation accuracy of centrifugal pump. J. Cent. South Univ. 2015, 22, 368-376. [CrossRef]

26. Slater, J. NPARC Alliance CFD Verification and Validation Web Site. J. Examining Spat. (Grid) Converg. 2021, 11, 513. 